\title{
Molecular identification and antifungal susceptibility patterns of dermatophytes isolated from companion animals with clinical symptoms of dermatophytosis
}

\author{
Farzad Katiraee ${ }^{1 凶}$, Yasaman Kouchak Kosari ${ }^{1}$, Minoo Soltani ${ }^{2}$, \\ Hojjatollah Shokri $^{3 凶}$, Mohammad Hassan Minooieanhaghighi ${ }^{4}$ \\ ${ }^{1}$ Department of Pathobiology, Faculty of Veterinary Medicine, University of Tabriz, Tabriz, Iran \\ ${ }^{2}$ Department of Microbiology, Faculty of Veterinary Medicine, University of Tehran, Tehran, Iran \\ ${ }^{3}$ Department of Pathobiology, Faculty of Veterinary Medicine, Amol University of Special Modern Technologies, Amol, Iran \\ ${ }^{4}$ Department of Basic Sciences, Faculty of Medicine, Gonabad University of Medical Sciences, Gonabad, Iran \\ f.katiraee@tabrizu.ac.ir, hshokri@ut.ac.ir
}

Received: May 11, $2019 \quad$ Accepted: March 24, 2021

\begin{abstract}
Introduction: Dermatophytosis is a common skin disease in cats and dogs caused by Microsporum and Trichophyton fungi. Species identification and knowledge of their antifungal susceptibility are therapeutically and epidemiologically important. This study assessed the prevalence of feline and canine dermatophytosis in Iran, identified the aetiological agents molecularly and tested their antifungal susceptibility. Material and Methods: A total of 308 companion animals (134 dogs and 174 cats) with skin lesions were examined from March 2015 to March 2018. Hair and skin samples were examined by microscopy with $20 \% \mathrm{KOH}$ and cultured on Sabouraud dextrose agar with cycloheximide and chloramphenicol. Fungal isolates were confirmed by sequencing of the internal transcribed spacer (ITS) r-DNA region. The antifungal susceptibility of dermatophytes was tested by broth microdilution assay using standard drugs. Results: Dermatophytes were found in $130(42.2 \%)$ samples, 62 of them feline and 68 canine. Based on sequencing of all strains, M. canis $(78.5 \%, \mathrm{P}<0.05), M$. gypseum $(10.7 \%)$, and T. mentagrophytes $(10.7 \%)$ were the dermatophytes isolated. The non-dermatophyte species Nannizziopsis vriesii was also isolated from two feline dermatomycosis cases. Dogs and cats younger than one year $(61.5 \%)$ showed a statistically significantly higher prevalence of infection $(\mathrm{P}<0.05)$. Caspofungin produced the lowest geometric mean MIC at $0.0018 \mu \mathrm{g} / \mathrm{mL}$, followed by ketoconazole, terbinafine, itraconazole, miconazole, griseofulvin, clotrimazole and fluconazole, in a $0.038-1.53 \mu \mathrm{g} / \mathrm{mL}$ range. Conclusion: This is the first molecular study to identify the causes of pet dermatophytosis in north-western Iran. ITS-PCR was shown to be a useful and reliable method for the identification of closely related species of dermatophytes in clinical and epidemiological settings. The lowest MIC of caspofungin indicated that this drug was the most potent in vitro.
\end{abstract}

Keywords: dermatophytosis, cat, dog, Microsporum canis, ITS-PCR, antifungals.

\section{Introduction}

Dermatophytosis is a superficial fungal infection of hair and keratinised layers of the epidermis and is caused by keratinophilic dermatophytes, such as Microsporum, Trichophyton and Epidermophyton (29). It is an endemic infection in many countries throughout the world affecting companion animals (dogs and cats), domestic animals (calves), and laboratory animals (rabbits) as well as humans $(17,26)$. Companion animals (dogs and cats) can act as carriers of Microsporum, which cannot invade healthy skin of these animals. This carrier stage may progress to infection based on certain predisposing factors, such as young age, immunosuppression, nutritional deficiency, high environmental temperature with high humidity, and skin trauma (34).

The gold standard diagnostic techniques for the identification of dermatophytosis involve direct microscopic examination of clinical samples followed by in vitro isolation and identification (30). In addition, the use of PCR to diagnose dermatophytes in lesions in dogs and cats confirmed its usefulness, given that this 
can be done via infected samples. There are limited published studies evaluating PCR-based methodology for the diagnosis of dermatophytosis from cat or dog hair $(9,16)$.

The antifungals commonly used in the systemic treatment of dermatophytosis in dogs and cats include itraconazole, terbinafine and griseofulvin. Currently, the emergence of antifungal-resistant clinical isolates can lead to failure in the treatment of mycosis $(1,23)$. Therefore, an in vitro antifungal susceptibility test could help to optimise the therapy and select an effective antifungal agent against the specific clinical isolates from an animal's dermatophytic samples.

A growing tendency to keep companion animals such as dogs and cats was observed in north-western Iran and these animals are very closely associated with the daily life of their owners, especially owners who are still children. Although there are some reports regarding the occurrence of dermatophytes in companion animals in the study area (24) and the application of PCR and antifungal assays on clinical dermatophytes from veterinary patients (28), these studies are few in number, were performed with a limited number of samples, and could not elucidate their antifungal susceptibility or the current trend of infection. Therefore, the present study was conducted, and it reports molecular dermatophyte identification, the antifungal susceptibility of these fungi, and the occurrence of the carrier state in infected companion animals (dogs and cats), which are considered the most potent carriers in Iran.

\section{Material and Methods}

Study population. A total of 308 companion animals, comprising 134 dogs and 174 cats in northwestern Iran were examined for evidence of dermatophytosis at the University of Tabriz, Iran, from March 2015 to March 2018. The skin of the animals was examined by a veterinarian for any lesions. The animals were male and female and were divided into three groups by age: group 1 were $0-12$ months, group 2 were 1-4 years and group 3 were over than 4 years old. The dogs were kept indoors and they often shared the floor, bed, or sofa with their owners and particularly the children in dog-owning families. However, the cats preferred to roam outside the house during the daytime.

Sampling. The sampling was carried out at the pet clinic of the University of Tabriz. A new toothbrush or hair brush was used on the animal's skin over the back, shoulders, sides, hindquarters and legs for 5-7 min and shed or abraded material was collected. Both the hair and skin scraping samples were wrapped in sterile Petri dishes and were kept in an airtight container with the minimum practical moisture level for transport to the mycological laboratory of the University of Tabriz. The collection procedure was approved by the Committee on Animal Ethics of the University of Tabriz and performed according to the International
Guiding Principles for Biomedical Research Involving Animals.

Direct examination. All samples were examined for fungal elements under a light microscope at $40 \times$ magnification after imbibition in $20 \%$ potassium hydroxide $(\mathrm{KOH})$.

Fungal culture. The hair and skin scraping samples were inoculated into Sabouraud dextrose agar containing chloramphenicol (SC) and Sabouraud dextrose agar containing chloramphenicol and cycloheximide (SCC). The plates were incubated at $30^{\circ} \mathrm{C}$ and examined daily for four weeks. Each of the fungal isolates was identified based on its colony characteristics and hyphal and conidial cells. The conidia were identified after lactophenol cotton blue staining on the basis of their size, shape, presence of septa, thickness of the conidial wall and arrangement of conidial cells around the hyphae. To confirm the identification of dermatophytes, the internal transcribed spacer (ITS)-PCR method relying on the ITS1-5.8S-ITS2 region was also performed as described by RezaeiMatehkolaei et al. (31).

Molecular identification of dermatophytes. For DNA extraction, genomic DNA was extracted by a physicochemical method (11). Mycelia ( $\sim 50 \mathrm{mg}$ wet weight) were harvested and ground by glass beads, then $500 \mu \mathrm{L}$ of lysis buffer ( $400 \mathrm{mM}$ of Tris- $\mathrm{HCl}(\mathrm{pH} 8.0)$, $60 \mathrm{mM}$ of EDTA ( $\mathrm{pH} \mathrm{8.0)}$, and $150 \mathrm{mM}$ of $\mathrm{NaCl}$, $1 \%$ sodium dodecyl sulphate) was added and the mixture was left at room temperature for $10 \mathrm{~min}$. Addition of $20 \mu \mathrm{L}$ of proteinase $\mathrm{K}(20 \mathrm{mg} / \mathrm{mL})$ to the tube followed and incubation for $1 \mathrm{~h}$ at $55^{\circ} \mathrm{C}$ and $10 \mathrm{~min}$ at $95^{\circ} \mathrm{C}$ in a water bath took place next. After adding $150 \mu \mathrm{L}$ of potassium acetate $5 \mathrm{M}$ ( $\mathrm{pH} 4.8$ with glacial acetic acid), the tube was vortexed briefly and centrifuged at 10,000 $\mathrm{g}$ for $2 \mathrm{~min}$. An equal volume of cold isopropyl alcohol was added to the supernatant and mixed by inversion spin at $12,000 \mathrm{~g}$ for $2 \mathrm{~min}$ and the supernatant was discarded. The DNA pellet was washed with $300 \mu \mathrm{L}$ of $70 \%$ ethanol, air dried and dissolved in $50 \mu \mathrm{L}$ of sterile water. Fungal spores and mycelia were disrupted with a freeze-thawing method with the aid of glass beads, and centrifuged at $10,000 \mathrm{~g}$ for $2 \mathrm{~min}$. A $500 \mu \mathrm{L}$ volume of lysis buffer was added to the precipitant and the solution was incubated at room temperature for $10 \mathrm{~min}$. Potassium acetate buffer $\mathrm{pH} 4.8$ was also added in a $150 \mu \mathrm{L}$ volume and the tube was vortexed briefly and cell debris and precipitated proteins were removed by centrifugation. The supernatant was transferred to another microtube and centrifuged. Then the supernatant was transferred to a new $1.5 \mathrm{~mL}$ microtube and an equal volume of isopropyl alcohol was added. The tube was mixed briefly by inversion, centrifuged at $12,000 \mathrm{~g}$ for 2 min and the supernatant was discarded. The resultant DNA pellet was washed three times in $300 \mu \mathrm{L}$ of $70 \%$ $(\mathrm{v} / \mathrm{v})$ ethanol. After centrifuging at $12,000 \mathrm{~g}$ for $1 \mathrm{~min}$, the supernatant was discarded. The DNA was dried and dissolved in $50 \mu \mathrm{L}$ of distilled water. In order to measure the concentration and purity of the DNA, the optical density (OD) was read in agarose gel. 
The ITS rDNA regions of all samples were amplified in a PCR by the universal fungal primers V9G (5'-TTACgTCCCTgCCCTTTgTA-3') and LS266 (5'-GCATTCCCAAACAACTCgACTC-3') (21, 30). Amplification was carried out by a PCR mixture containing $5 \mu \mathrm{L}$ of $10 \mathrm{x}$ reaction buffer, $200 \mu \mathrm{M}$ of dNTPs mixture, $0.25 \mu \mathrm{L}$ of Taq polymerase $(5 \mathrm{U} / \mu \mathrm{L})$, 30 pmol of each forward and reverse primer, $2 \mu \mathrm{L}$ of DNA template solution and ultrapure water up to a final volume of $50 \mu \mathrm{L}$. Each reaction mixture was preheated to $94^{\circ} \mathrm{C}$ for $5 \mathrm{~min}$, then the PCR was performed under the following protocol: 35 cycles of $30 \mathrm{~s}$ at $94^{\circ} \mathrm{C}, 30 \mathrm{~s}$ at $58^{\circ} \mathrm{C}$ and $1 \mathrm{~min}$ at $72^{\circ} \mathrm{C}$; a final extension at $72^{\circ} \mathrm{C}$ for $5 \mathrm{~min}$ and cooling at $4^{\circ} \mathrm{C}$. Sequence analysis was performed by comparison of the test nucleotide sequences with reference dermatophyte nucleotide sequences obtained from the Central Bureau of Fungal Cultures database at the Westerdijk Fungal Biodiversity Institute (http://www.cbs.knaw.nl/dermatophytes/Biolo MICSID.aspx). Similarity of $>99 \%$ to the reference ITS sequences was revealed. Accession numbers were also obtained from GenBank for the dermatophyte sequences (https://www.ncbi.nlm.nih. gov/genbank/).

Antifungal susceptibility testing. The antifungal susceptibility of clinical dermatophytes was tested by broth microdilution assay according to the Clinical Laboratory Standards Institute (CLSI) approved standard M38-A2 (13) using fluconazole, ketoconazole, itraconazole, miconazole, clotrimazole, griseofulvin, caspofungin and terbinafine (Sigma Chemical Corporation, St. Louis, MO, USA). Briefly, 10 two-fold drug dilutions were prepared in Roswell Park Memorial Institute-1640 (RPMI-1640) medium from stock solutions in a 96-well microtitre plate. Inoculum suspensions of dermatophytes were prepared in sterile saline from 7-10 day old SCC slants that had been incubated at $30^{\circ} \mathrm{C}$. Hyphal fragments and conidia were harvested with sterile wet swabs in saline, vortexed for $20 \mathrm{~s}$ and then kept at room temperature for 15-20 min to enable heavy hyphal fragments and conidia to settle. Homogenous suspensions of the supernatant were collected in new sterile tubes, adjusted to $0.11 \mathrm{OD}$ at $530 \mathrm{~nm}$ and diluted 1:50 in the RPMI-1640 media to achieve double concentrated inoculum suspensions of $0.5 \times 10^{4}-4.0 \times 10^{4} \mathrm{CFU} / \mathrm{mL}$. The concentrations were confirmed by counting the conidia in a haemocytometer and counting colonies after plating serial dilutions of the inoculum suspensions on SCC medium. Microdilution plates were set up in accordance with the CLSI M38-A2 standard. Column 1 was filled with $200 \mu \mathrm{L}$ of RPMI-1640 medium without drug or inoculum suspension, to serve as a sterility control. Double-sized inoculum suspensions of $100 \mu \mathrm{L}$ volume were added to columns 2 to 11 , which already contained $100 \mu \mathrm{L}$ of serially diluted antifungal agents. Column 12 contained $100 \mu \mathrm{L}$ of the inoculum and $100 \mu \mathrm{L}$ of drug-free RPMI-1640 medium to serve as growth controls. The microtitre plates were incubated at $30^{\circ} \mathrm{C}$ and read after a minimum of 4 days' incubation. In each experiment, two quality control strains, Candida albicans ATCC 10231 and Candida parapsilosis ATCC 22019, were included. Minimum inhibitory concentrations 50 and $90\left(\mathrm{MIC}_{50}\right.$ and $\left.\mathrm{MIC}_{90}\right)$ were defined as the points at which there was $50 \%$ and $90 \%$ inhibition of growth as compared with the growth control when read visually in microtitre plates.

Statistical analysis. The chi-square $\left(X^{2}\right)$ test was used to assess statistical differences between the groups. A P value less than 0.05 was statistically considered significant.

\section{Results}

Of the 308 examined scrapings, 126 (40.9\%) were positive for fungal elements by direct microscopic examination. There were 130 (42.2\%) culture-positive samples among them. Four (1.2\%) samples considered negative upon direct microscopic examination were positive for dermatophytes in culture. The comparison of the results of direct microscopic examination and fungal culture is summarised in Table 1. Sixty two (46.2\%) of the 134 dogs and $68(39.1 \%)$ of the 174 cats were determined positive for dermatophytes.

As shown in Table 2, the only risk factor found to be significantly associated with dermatophytosis was age. Dogs and cats in their first year of life (61.5\%) showed a statistically significant higher prevalence of infection than older animals $(\mathrm{P}<0.05)$. Regarding gender, 72 cases $(55.4 \%)$ were female and 58 cases (44.6\%) were male, indicating no significant difference in the incidence of dermatophytosis between the two sexes (Table 2).

The amplicons obtained for dermatophyte species using the V9G/LS266 primer were shown in Fig.1.

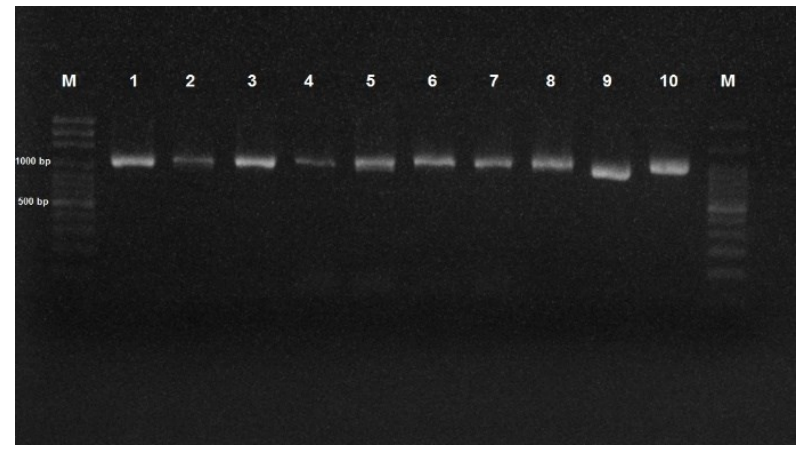

Fig 1. Representative amplified ITS region using V9G-LS266 primer. Lines 1-8-M. canis; line 9-M. gypseum; line10-T. mentagrophytes; $\mathrm{M}-100$ bp DNA ladder

Based on PCR and sequence analysis of the ITS region, the most frequently isolated dermatophyte was $M$. canis (78.4\%), followed by M. gypseum (10.8\%) and T. mentagrophytes (10.8\%) (Table 3). Microsporum canis was the most common dermatophyte isolated from dogs $(74.3 \%, \mathrm{P}<0.05)$, followed by $T$. mentagrophytes (16\%). ITS sequences were submitted to GenBank and deposited under the accession numbers KY070120- 
KY070141. However, no significant difference in prevalence was found among other dermatophyte species $(\mathrm{P}>0.05)$. The same difference in species prevalence as emerged in dogs was detected among species isolated from cats $(\mathrm{P}<0.05)$, where $M$. canis also had the highest isolation rate $(82.3 \%)$. The prevalence of T. mentagrophytes was 2.5 -fold greater in dogs than in cats. The dermatophyte species isolated and their prevalence in dogs and cats are shown in Table 3.

Nannizziopsis vriesii as a non-dermatophytic species was isolated from two feline cases with dermatomycosis. It is noteworthy that most of the T. mentagrophytes as well as $N$. vriesii were isolated from animals that were kept in outdoor and indoor conditions. The other fungal genera isolated were Malassezia and Candida (Table 4). A significant difference was observed between the prevalence of dermatophytic and non-dermatophytic fungi $(\mathrm{P}<0.05)$.
In vitro activities of eight antifungal agents that potentially can be used either orally or topically, following microdilution and 7-day incubation at $30^{\circ} \mathrm{C}$ are presented in Table 5. Geometric mean (GM) MICs, MIC range, $\mathrm{MIC}_{50}$ and $\mathrm{MIC}_{90}$ were obtained for all the isolates tested. When all the strains were considered together, caspofungin produced the lowest GM MICs $(0.0018 \mu \mathrm{g} / \mathrm{mL})$, followed by ketoconazole $(0.038 \mu \mathrm{g} / \mathrm{mL})$, terbinafine $(0.043 \mu \mathrm{g} / \mathrm{mL})$, itraconazole $(0.073 \mu \mathrm{g} / \mathrm{mL})$, miconazole $(0.15 \mu \mathrm{g} / \mathrm{mL})$, griseofulvin $(0.36 \mu \mathrm{g} / \mathrm{mL})$, clotrimazole $(0.61 \mu \mathrm{g} / \mathrm{mL})$ and fluconazole $(1.53 \mu \mathrm{g} / \mathrm{mL})$. The lowest $\mathrm{MIC}_{50}$ and $\mathrm{MIC}_{90}$ values of caspofungin were found to be 0.001 and $0.002 \mu \mathrm{g} / \mathrm{mL}$ for M. canis, followed by 0.002 and $0.004 \mu \mathrm{g} / \mathrm{mL}$ for $T$. mentagrophytes and 0.002 and $0.008 \mu \mathrm{g} / \mathrm{mL}$ for $M$. gypseum, indicating that the first drug was the most potent in the in vitro study.

Table 1. Results of direct microscopic examinations versus those of cultures

\begin{tabular}{lllr}
\hline & Test results & Dog $(\mathrm{n}=134)$ & $\begin{array}{c}\text { Animal } \\
\text { Cat }(\mathrm{n}=174)\end{array}$ \\
\hline \multirow{2}{*}{ Microscopy } & Positive & $62(46.2 \%)$ & $64(38.4 \%)$ \\
\cline { 2 - 4 } & Negative & $72(53.8 \%)$ & $110(61.6 \%)$ \\
\hline \multirow{nyyy}{*}{ Culture } & Positive & $62(46.2 \%)$ & $68(39.1 \%)$ \\
\hline
\end{tabular}

Table 2. Profiles of animals with suspected cases of dermatophytosis

\begin{tabular}{|c|c|c|c|c|}
\hline & & \multicolumn{2}{|c|}{ Animal } & \multirow{2}{*}{ Total $(n=130)$} \\
\hline & & $\operatorname{Dog}(n=62)$ & Cat $(n=68)$ & \\
\hline \multirow{3}{*}{ Age } & $<1$ year old & $36(58.1 \%)$ & $44(64.7 \%)$ & $80(61.5 \%)$ \\
\hline & $1-4$ years old & $10(16.1 \%)$ & $16(23.5 \%)$ & $26(20 \%)$ \\
\hline & $>4$ years old & $16(25.8 \%)$ & $8(11.7 \%)$ & $24(18.5 \%)$ \\
\hline \multirow{2}{*}{ Sex } & Female & $26(41.9 \%)$ & $46(67.6 \%)$ & $72(55.4 \%)$ \\
\hline & Male & $36(58.1 \%)$ & $22(32.4 \%)$ & $58(44.6 \%)$ \\
\hline \multirow{2}{*}{ Habitat } & Indoor & $50(80.6 \%)$ & $34(50 \%)$ & $84(64.6 \%)$ \\
\hline & Outdoor & $12(19.4 \%)$ & $34(50 \%)$ & $46(35.4 \%)$ \\
\hline \multirow{2}{*}{ Disease in owner } & Yes & $8(12.9 \%)$ & $18(26.5 \%)$ & $26(25 \%)$ \\
\hline & No & $54(87.1 \%)$ & $50(73.5 \%)$ & $104(65 \%)$ \\
\hline \multirow{2}{*}{$\begin{array}{l}\text { Previous antifungal } \\
\text { therapy for cutaneous } \\
\text { lesions }\end{array}$} & Yes & $4(6.5 \%)$ & $6(8.8 \%)$ & $10(7.7 \%)$ \\
\hline & No & $58(93.5 \%)$ & $62(91.2 \%)$ & $120(92.3 \%)$ \\
\hline
\end{tabular}

Table 3. Results of ITS sequence analysis of canine and feline dermatophyte samples

\begin{tabular}{|c|c|c|c|c|}
\hline \multirow{2}{*}{\multicolumn{2}{|c|}{ Molecular results }} & \multicolumn{2}{|c|}{ Animal } & \multirow{2}{*}{ Total $(n=130)$} \\
\hline & & $\operatorname{Dog}(n=62)$ & Cat $(n=68)$ & \\
\hline \multirow{3}{*}{ ITS sequenced analysis } & M. canis & $46(74.3 \%)$ & $56(82.3 \%)$ & $102(78.4 \%)$ \\
\hline & T. mentagrophytes & $10(16 \%)$ & $4(5.9 \%)$ & $14(10.8 \%)$ \\
\hline & M. gypseum & $6(9.7 \%)$ & $8(11.8 \%)$ & $14(10.8 \%)$ \\
\hline
\end{tabular}


Table 4. Fungal strains isolated from animals with skin lesions suspected of being dermatophytes

\begin{tabular}{lllll}
\hline Genus & Fungal species & Dog & Cat & Total \\
\hline $\begin{array}{l}\text { Microsporum } \\
\text { Trichophyton } \\
\text { (Dermatophytes) }\end{array}$ & M. canis & 46 & 56 & 102 \\
& T. mentagrophytes & 10 & 4 & 14 \\
& M. gypseum & 6 & 8 & 48 \\
Malassezia & M. pachydermatis & 22 & 2 & 4 \\
& M. furfur & 2 & 3 & 4 \\
M. globosa & 1 & 0 & 3 \\
Candida & Calbicans & 3 & 0 & 2 \\
\hline
\end{tabular}

Table 5. In vitro antifungal susceptibilities of eight antifungal drugs against 130 clinical isolates of dermatophytes from dogs and cats with dermatophytosis

\begin{tabular}{|c|c|c|c|c|c|}
\hline Dermatophyte species & Drug & MIC range $(\mu \mathrm{g} / \mathrm{mL})$ & $\begin{array}{l}\mathrm{GM} \\
(\mu \mathrm{g} / \mathrm{mL})\end{array}$ & $\begin{array}{l}\mathrm{MIC}_{50} \\
(\mu \mathrm{g} / \mathrm{mL})\end{array}$ & $\begin{array}{l}\mathrm{MIC}_{90} \\
(\mu \mathrm{g} / \mathrm{mL})\end{array}$ \\
\hline \multirow{8}{*}{$\begin{array}{l}\text { Microsporum canis } \\
(\mathrm{n}=102)\end{array}$} & Caspofungin & $0.001-0.008$ & 0.001 & 0.001 & 0.002 \\
\hline & Ketoconazole & $0.032-8$ & 0.064 & 0.032 & 0.125 \\
\hline & Miconazole & $0.032-0.5$ & 0.12 & 0.250 & 0.250 \\
\hline & Itraconazole & $0.002-2$ & 0.06 & 0.125 & 0.25 \\
\hline & Griseofulvin & $0.125-8$ & 0.6 & 1 & 1 \\
\hline & Clotrimazole & $0.016-16$ & 0.12 & 1 & 2 \\
\hline & Fluconazole & $0.128-32$ & 2.1 & 4 & 8 \\
\hline & Terbinafine & $0.008-2$ & 0.064 & 0.125 & 0.25 \\
\hline \multirow{8}{*}{$\begin{array}{l}\text { Trichophyton } \\
\text { mentagrophytes }(\mathrm{n}=14)\end{array}$} & Caspofungin & $0.001-0.016$ & 0.0025 & 0.002 & 0.004 \\
\hline & Ketoconazole & $0.016-0.128$ & 0.026 & 0.016 & 0.032 \\
\hline & Miconazole & $0.064-0.5$ & 0.2 & 0.128 & 0.5 \\
\hline & Itraconazole & $0.002-1$ & 0.09 & 0.125 & 0.25 \\
\hline & Griseofulvin & $0.128-2$ & 0.25 & 0.250 & 1.0 \\
\hline & Clotrimazole & $0.5-8$ & 0.9 & 0.5 & 4 \\
\hline & Fluconazole & $0.5-4$ & 1.41 & 1 & 4 \\
\hline & Terbinafine & $0.016-0.125$ & 0.026 & 0.064 & 0.064 \\
\hline \multirow{8}{*}{$\begin{array}{l}\text { Microsporum gypseum } \\
(\mathrm{n}=14)\end{array}$} & Caspofungin & $0.001-0.004$ & 0.0019 & 0.002 & 0.008 \\
\hline & Ketoconazole & $0.016-0.064$ & 0.026 & 0.016 & 0.064 \\
\hline & Miconazole & $0.064-0.5$ & 0.15 & 0.125 & 0.5 \\
\hline & Itraconazole & $0.002-0.5$ & 0.07 & 0.125 & 0.25 \\
\hline & Griseofulvin & $0.064-2$ & 0.23 & 0.125 & 1.0 \\
\hline & Clotrimazole & $0.25-4$ & 0.82 & 0.5 & 4 \\
\hline & Fluconazole & $0.25-4$ & 1.1 & 1 & 2 \\
\hline & Terbinafine & $0.016-0.064$ & 0.039 & 0.016 & 0.064 \\
\hline
\end{tabular}

GM - geometric mean

\section{Discussion}

Dermatophytosis constitutes a major public health problem in several countries. The most common factors affecting the distribution and transmission of dermatophytic infections are animal contact, general hygiene and climatic conditions (30). This study highlighted the distribution of dermatophytes in dogs and cats with suspected dermatophytosis, the prevalence of infection in relation to sex and age, molecular diagnosis of dermatophytes and their susceptibility patterns.

In the present study, dermatophytes were isolated from $42.2 \%$ of dogs and cats with suspected lesions of dermatophytosis. The fungal cultures were positive in 
$46.2 \%$ and $39.1 \%$ of canine and feline samples. Previous studies showed different isolation rates of dermatophytes in $\operatorname{dog} s$ and cats suspected with dermatophytosis in different world regions. Respectively for dogs and cats, positive cultures were reported in $13 \%$ and $34 \%$ of samples in Spain (8), in $21 \%$ and $28 \%$ in Italy (10), in $18.2 \%$ and $51.9 \%$ in Greece (27), and in $33 \%$ and $35 \%$ in Brazil (7). There are few studies on the epidemiology of animal dermatophytosis in Iran. In a study conducted by Khosravi and Mahmoudi (25), 55\% of cats' samples and $9 \%$ of dogs' samples were positive for dermatophytes. Yahyaraeyat et al. (35) showed that 54\% and $22 \%$ of suspected cats and dogs were positive for dermatophytes, respectively. In a retrospective study by Shokri and Khosravi (34), there were $49.7 \%$ positive results, of which $87.8 \%$ and $24.3 \%$ were associated with infected cats and dogs, respectively. In agreement with our results, Seker and Dogan found positivity only $1 \%$ higher in dogs' samples than cats' (33), but different studies throughout the world have shown that cats were more sensitive. In fact, our study included only samples from animals with skin lesions such as alopecia and desquamation whereas asymptomatic animals, especially cats without lesions, have key roles in the spreading and epidemiology of dermatophytosis among animals and humans. Therefore, the higher isolation rate of dermatophytes from dogs than cats in the present study may not reflect the true prevalence of dermatophytosis in these areas of Iran.

Dermatophytosis occurs at all ages, but it is more common in young animals. Our results also proved this fact, because $61.5 \%$ of dogs and cats less than one year of age showed a statistically significant higher prevalence of infection than older animals. In agreement with our results, there were similar studies that support this claim, such as those by Cafarchia et al. (10), Gangil et al. (20), Patel (29) and Shokri and Khosravi (34).

The present study showed that from 130 positive cases, $55.4 \%$ were female and $44.6 \%$ male. Although some investigators reported that male dogs and cats were more often affected (10), several researchers did not observe any correlation between sex and the presence of dermatophytosis $(9,17)$. In our study, similar to other reports, no statistically significant association was found between the sex of dogs and cats and the presence of dermatophytosis, indicating that it did not play a role in an animal's propensity to suffer the disease.

Accurate identification of fungal agents is a basic step in epidemiological studies necessary in order to understand new pathogenic fungi and design treatment. Dermatophytes are very similar and closely related to each other, so various physiological and phenotypical methods are essential for accurate identification. These methods are often time consuming and cannot be repeated, and also sometimes they do not lead to accurate identification. Now, sequencing of rDNA is the standard method for the identification of most fungi, such as dermatophytes, and is proven to be reliable. Identification by sequencing exploits the aptness of the
ITS1-5.8s-ITS2 region for the determination of most dermatophytic species $(22,30)$.

The present study isolated M. canis, M. gypseum and $T$. mentagrophytes from dogs and cats with dermatophytosis. The dermatophyte species identified in this research are similar to the species isolated from cats and dogs from different regions reported by Khosravi and Mahmoudi (25), Chermette et al. (12), Beraldo et al. (7), and Shokri and Khosravi (34). Microsporum canis was the most frequently isolated dermatophyte from companion cats and dogs, which is in agreement with earlier findings throughout the world $(6,14,33)$. However, there were significant differences between our results and those of other studies $(20,32)$. The use of a molecular technique for dermatophyte identification in the present research and geographical variations may be the reasons for the discrepancy in the distribution of species compared to the results of other studies. Due to the improvements in rapid molecular identification of fungal pathogenic species, these methods should be considered for routine use, as their more extensive employment would lead to more accurate results and more diverse species recognition.

In this study, we employed the broth microdilution method to determine the MICs of antifungal agents for clinical dermatophytes. The antibiograms for 130 dermatophytes showed that their highest susceptibility was to caspofungin and the lowest to fluconazole. There was no significant difference in patterns of susceptibility among dermatophytic species $(\mathrm{P}>0.05)$. The results showed that for all tested isolates, in terms of $\mathrm{MIC}_{50}$ and $\mathrm{MIC}_{90}$, caspofungin $(0.002 \mu \mathrm{g} / \mathrm{mL}$ and $0.005 \mu \mathrm{g} / \mathrm{mL})$ was more active than the other tested drugs. The in vitro efficacy of echinocandin drugs, especially caspofungin, against dermatophytes was first evaluated by Bao et al. (5), who demonstrated that caspofungin exhibited good in vitro antifungal activity to dermatophytes and induced microscopic morphological changes in hyphae. Recent studies also showed that caspofungin has potent in vitro activity against dermatophytes, although the relevance for clinical efficacy has not yet been established (3, 4, 18). The absence of in vivo studies of echinocandin efficacy limits their use for the treatment of dermatophytosis, which may underlie the absence of resistant dermatophyte strains described to date. Globally, the $\mathrm{MIC}_{50}$ and $\mathrm{MIC}_{90}$ reported for itraconazole, miconazole, ketoconazole, terbinafine and griseofulvin to dermatophytes were found to be generally low $(<1 \mu \mathrm{g} / \mathrm{mL})(2,19)$. However, there were a few species-specific studies where high MIC values were reported for fluconazole $(>1-32 \mu \mathrm{g} / \mathrm{mL})(1,15,23$, 32). Similarly, our antifungal results showed high MIC values of $>1 \mu \mathrm{g} / \mathrm{mL}$ for fluconazole against almost $100 \%$ of clinical isolates. The authors of the referenced studies clarify that the clinical significance of these high MICs is unclear, as patient outcomes were not followed up, and there is a general lack of studies correlating dermatophyte antifungal MICs with treatment outcomes. 
Our study provided useful insights into the reliability of the ITS-PCR method for the identification of dermatophytes. The most frequent dermatophyte isolated from infected dogs and cats was M. canis. The present research also provided useful information regarding the antifungal susceptibility patterns of clinical dermatophytes and demonstrated low MIC values of caspofungin and high MIC values of fluconazole to our isolates. To prevent the unnecessary usage of toxic drugs, regular surveillance of antifungal susceptibility patterns in infected animals should be carried out. This is the first molecular epidemiological study of feline and canine dermatophytosis in the north-west of Iran and affords knowledge of the epidemiology of the disease in companion animals, which is essential to reduce the spread of zoophilic fungal infections to humans.

Conflict of Interests Statement: The authors declare that there is no conflict of interests regarding the publication of this article.

Financial Disclosure Statement: The current study was supported by the Research Council of the University of Tabriz, Tabriz, Iran.

Animal Rights Statement: The collection proceedings were approved by the Committee on Animal Ethics of the University of Tabriz and performed according to the International Guiding Principles for Biomedical Research Involving Animals.

\section{References}

1. Abastabar M., Jedi A., Guillot J., Ilkit M., Eidi S., Hedayati M.T., Shokohi T., Daie Gazvini R., Rezaie-Matehkolaei A., Katiraee F., Javidnia J., Ahmadi B., Badali $\mathrm{H}$ : In vitro activities of 15 antifungal drugs against a large collection of clinical isolates of Microsporum canis. Mycoses 2019, 62, 1069-1078, doi: 10.1111/myc. 12986 .

2. Araújo C.R., Miranda K.C., Fernandes O.F.L., Soares A.J., Silva M.R.R.: In vitro susceptibility testing of dermatophytes isolated in Goiania, Brazil, against five antifungal agents by broth microdilution method. Rev Inst Med Trop 2009, 51, 9-12, doi: 10.1590/s0036-46652009000100002.

3. Badali H., Mohammadi R., Mashedi O., de Hoog G.S., Meis J.F.: In vitro susceptibility patterns of clinically important Trichophyton and Epidermophyton species against nine antifungal drugs. Mycoses 2015, 58, 303-307, doi: 10.1111/myc.12315.

4. Baghi N. Shokohi T., Badali H., Makimura K., Rezaei-Matehkolaei A., Abdollahi M., Didehar M., Haghani I., Abastabar M.: In vitro activity of new azoles luliconazole and lanoconazole compared with ten other antifungal drugs against clinical dermatophyte isolates. Med Mycol 2016, 54, 757-763, doi: 10.1093/mmy/ myw016.

5. Bao YQ., Wan Z., Li RY.: In vitro antifungal activity of micafungin and caspofungin against dermatophytes isolated from China. Mycopathologia 2013, 175, 141-145, doi: 10.1007/ s11046-012-9571-6.

6. Beigh S.A., Soodan J.S., Singh R., Khan A.M., Dar M.A.: Evaluation of trace elements, oxidant/antioxidant status, vitamin $\mathrm{C}$ and $\beta$-carotene in dogs with dermatophytosis. Mycoses 2014, 57, 358-365, doi: $10.1111 /$ myc. 12163 .
7. Beraldo R.M., Gasparoto A.K., de Siqueira A.M., Tranches Dias A.L.: Dermatophytes in household cats and dogs. Rev Bras Ciência Vet 2011, 18, 85-91, doi: 10/4322/rbcv.2014.125.

8. Cabañes F.J., Abarca M.L., Bragulat M.R.: Dermatophytes isolated from domestic animals in Barcelona, Spain. Mycopathologia 1997, 137, 107-113, doi: 10.1023/a:1006867413987.

9. Cafarchia C., Gasser R.B., Figueredo L.A., Weigl S., Danesi P., Capelli G., Otranto D.: An improved molecular diagnostic assay for canine and feline dermatophytosis. Med Mycol 2013, 51, 136-143, doi: 10.3109/13693786.2012.691995.

10. Cafarchia C., Romito D., Sasanelli M., Lia R., Capelli G., Otranto D.: The epidemiology of canine and feline dermatophytoses in southern Italy. Mycoses 2004, 47, 508-513, doi: 10.1111/j.1439-0507.2004.01055.x.

11. Chander J.: Textbook of Medical Mycology, 3rd ed. Jaypee Brothers Medical Publishers, New Delhi, 2009.

12. Chermette R., Ferreiro L., Guillot J.: Dermatophytoses in animals. Mycopathologia 2008, 166, 385-405, doi: 10.1007/s11046-0089102-7.

13. Clinical Laboratory Standards Institute: M38-A2 Reference Method for Broth Dilution Antifungal Susceptibility Testing of Filamentous Fungi; Approved Standard, Second Edition. CLSI, Wayne, 2008.

14. da Costa F.V., Farias M.R., Bier D., de Andrade C.P., de Castro L.A., da Silva S.C., Ferreiro L.: Genetic variability in Microsporum canis isolated from cats, dogs and humans in Brazil. Mycoses 2013, 56, 582-588, doi: 10.1111/myc.12078.

15. da Silva Barros M.E., Assis Santos D., Hamdan J.S.: In vitro methods for antifungal susceptibility testing of Trichophyton spp. Mycological Res 2006, 110, 1355-1360, doi: 10.1016/j.mycres. 2006.08.006.

16. Dąbrowska I., Dworecka-Kaszak B., Brillowska-Dąbrowska A.: The use of a one-step PCR method for the identification of Microsporum canis and Trichophyton mentagrophytes infection of pets. Acta Biochim Pol 2014, 61, 375-378.

17. Debnath C., Mitra T., Kumar A., Samanta I.: Detection of dermatophytes in healthy companion dogs and cats in eastern India. Iran J Vet Res 2016, 17, 20-24.

18. Deng S.W., Ansari S., Ilkit M., Rafati H., Hedayati M.T., Taghizadeh-Armaki M., Nasrollahi-Omran A., Tolooe A., Zhan P., Liao W., van der Lee H.A., Verweij P.E., Seyedmousavi S.: In vitro antifungal susceptibility profiles of 12 antifungal drugs against 55 Trichophyton schoenleinii isolates from tinea capitis favosa patients in Iran, Turkey, and China. Antimicrob Agents Chemother 2017, 61, e01753-16, doi: 10.1128/AAC.01753-16.

19. Gadangi I.: In vitro antifungal susceptibility testing of 5 antifungal agents against dermatophytic species by CLSI (M38-A) micro dilution method. Clin Microbiol 2014, 3, 145, doi: 10.4172/23275073.1000145 .

20. Gangil R., Dutta P., Tripathi R., Singathia R.: Incidence of dermatophytosis in canine cases presented at Apollo Veterinary College, Rajashtan, India. Vet World 2012, 5, 682-684, doi: 10.5455/vetworld.2012.682-684.

21. Garces G.H., Hrycyk M.F., Giacobino J., Capela Machado G., Domingos Arantes T., Theodoro R.C., de M.G. Bosco S., Bagagli E.: Molecular identification and phylogenetical analysis of dermatophyte fungi from Latin America. Mycoses 2016, 59, 787-797, doi: 10.1111/myc.12532.

22. Garg J., Tilak R., Garg A., Prakash P., Gulati A.K., Nath G.: Rapid detection of dermatophytes from skin and hair. BMC Res Notes 2009, 2, 60, doi: 10.1186/1756-0500-2-60.

23. Gupta A.K., Kohli Y.: In vitro susceptibility testing of ciclopirox, terbinafine, ketoconazole and itraconazole against dermatophytes and nondermatophytes, and in vitro evaluation of combination antifungal activity. Br J Dermatol 2003, 149, 296-305, doi: 10.1046/j.1365-2133.2003.05418.x.

24. Katiraee F., Asharafi Helan J., Teifoori F.: Multiple cases of feline dermatophytosis due to Microsporum canis transmitted to their owners. J Zoonotic Dis 2016, 1, 24-27. 
25. Khosravi A.R., Mahmoudi M.: Dermatophytes isolated from domestic animals in Iran. Mycoses 2003, 46, 222-225, doi: 10.1046/j.1439-0507.2003.00868.x

26. Lund A., Bratberg A.M., Næss B., Gudding R.: Control of bovine ringworm by vaccination in Norway. Vet Immunol Immunopathol 2014, 158, 37-45, doi: 10.1016/j.vetimm.2013.04.007.

27. Menelaos L.A.: Dermatophytosis in dog and cat. Bulletin USAMV-CN 2006, 63, 304-308.

28. Nardoni S., Franceschi A., Mancianti F.: Identification of Microsporum canis from dermatophytic pseudomycetoma in paraffin-embedded veterinary specimens using a common PCR protocol. Mycoses 2007, 50, 215-217, doi: 10.1111/j.14390507.2007.01368.x.

29. Patel S., Meixner J.A., Smith M.B., McGinnis M.R.: Superficial Mycoses and Dermatophytes, In: Tropical Dermatology, edited by: S.K. Tyring, O. Lupi, U.R. Hengge, Elsevier, Amsterdam, 2017, pp. 189-201, doi: 10.1016/B978-0-323-29634-2.00017-1.

30. Petrucelli M.F., Abreu M.H., Cantelli B.A., Segura G.G., Nishimura F.G., Bitencourt T.A., Marins M., Fachin A.L. Epidemiology and Diagnostic Perspectives of Dermatophytoses. J Fungi 2020, 6, 310, doi: 10.3390/jof6040310.
31. Rezaei-Matehkolaei A., Jahangiri A., Mahmoudabadi A.Z., Najafzadeh M.J., Nouripour-Sisakht S., Makimura K.: Morphomolecular characterization of soil inhabitant dermatophytes from Ahvaz, Southwest of Iran, a high occurrence of Microsporum fulvum. Mycopathologia 2017, 182, 691-699, doi: 10.1007/s11046-017-0116-x.

32. Sarifakioglu E., Seçkin D., Demirbilek M., Can F.: In vitro antifungal susceptibility pattern of dermatophyte strains causing tinea unguium. Clin Exp Dermatol 2007, 32, 675-679, doi: 10.1111/j.1365-2230.2007.02480.x.

33. Seker E., Dogan N.: Isolation of dermatophytes from dogs and cats with suspected dermatophytosis in Western Turkey. Prev Vet Med 2011, 98, 46-51, doi: 10.1016/j.prevetmed.2010.11.003.

34. Shokri H., Khosravi A.R.: An epidemiological study of animals dermatomycoses in Iran. J Mycol Med 2016, 26, 170-177, doi: 10.1016/j.mycmed.2016.04.007.

35. Yahyaraeyat R., Shokri H., Khosravi A.R., Soltani M., Erfanmanesh A., Nikaein D.: Occurrence of animals dermatophytosis in Tehran, Iran. World J Zool 2009, 4, 200-204. 